\title{
Well-posedness of the Gnedenko System with Multiple Delay Vacations of a Repairman
}

\author{
Mahsut Rozi, Abdukerim Haji* and Abdugeni Osman \\ College of Mathematics and System Sciences, Xinjiang University, Urumqi 830046, China \\ ${ }^{*}$ Corresponding author
}

\begin{abstract}
The Gnedenko system with multiple delay vacations of a repairman is investigated. The well-posedness and the existence of the unique positive dynamic solution of the system are proved by using $C_{0}$-semigroup theory of linear operators in the functional analysis.
\end{abstract}

Keywords-gnedenko system; C_0-semigroup; dynamic solution

\section{INTRODUCTION}

The Gnedenko system is an important system in reliability theory. Since the strong practical background of The Gnedenko system, many researchers have studied it extensively under varying assumptions on the failures and repairs, see [1-5]. The repairman leaves for a vacation or does other work when there are no failed components for repair in system, which can have important influence to performance of system. In [5], the authors studied the Gnedenko system with multiple delay vacations of a repairman and obtained some reliability expressions such as the Laplace transform of the reliability, the mean time to the first failure, the availability and the failure frequency of the system. In [5], the authors used the dynamic solution in calculating the availability and the reliability. But they did not discuss the well-posedness and the existence of the positive dynamic solution. Motivated by this, we study in this paper the well-posedness and the existence of a unique positive dynamic solution of the system, by using $C_{0}$-semigroup theory of linear operators.

Gnedenko system with multiple delay vacations of a repairman can be described by the following equations (see [5]).

$$
(\mathrm{GS})\left\{\begin{array}{l}
\frac{d p_{0 d}(t)}{d t}=-\left(N \lambda+\lambda_{1}+\theta\right) p_{0 d}(t)+\int_{0}^{+\infty} p_{0 v}(t, y) r(y) d y \\
\quad+\int_{0}^{+\infty} p_{1 r}(t, x) \mu(x) d x \\
\frac{\partial p_{1 v}(t, y)}{\partial t}+\frac{\partial p_{1 v}(t, y)}{\partial y}=-\left(N \lambda+\lambda_{1}+r(y)\right) p_{0 v}(t, y) \\
\frac{\partial p_{0 v}(t, y)}{\partial t}+\frac{\partial p_{0 v}(t, y)}{\partial y}=-(N \lambda+r(y)) p_{1 v}(t, y) \\
+\left(N \lambda+\lambda_{1}\right) p_{0 v}(t, y) \\
\frac{\partial p_{1 v}(t, x)}{\partial t}+\frac{\partial p_{1 r}(t, x)}{\partial x}=-(N \lambda+\mu(x)) p_{1 r}(t, x) \\
\frac{\partial p_{2 v}(t, y)}{\partial t}+\frac{\partial p_{2 v}(t, y)}{\partial y}=-r(y) p_{2 v}(t, y)+N \lambda p_{2 v}(t, y) \\
\frac{\partial p_{2 r}(t, x)}{\partial t}+\frac{\partial p_{2 r}(t, x)}{\partial x}=-\mu(x) p_{2 r}(t, x)+N \lambda p_{1 r}(t, x)
\end{array}\right.
$$

with the boundary conditions

$$
(B C)\left\{\begin{aligned}
p_{0}(t, 0)= & \theta p_{o d}(t) \\
p_{1 r}(t, 0)= & \left(N \lambda+\lambda_{1}\right) p_{o d}(t)+\int_{0}^{+\infty} p_{1 v}(t, y) r(y) d y \\
& +\int_{0}^{+\infty} p_{2 r}(t, x) \mu(x) d x \\
p_{2 r}(t, 0)= & \int_{0}^{+\infty} p_{2 v}(t, y) r(y) d y \\
p_{1 v}(t, 0)= & p_{2 v}(t, o)=0
\end{aligned}\right.
$$

and the initial conditions

(IC) $\left\{\begin{array}{l}p_{0 d}(0)=1, \\ p_{0 v}(0, y)=p_{1 r}(0, x)=p_{1 v}(0, y)=p_{2 v}(0, y)=p_{2 r}(o, x)=0\end{array}\right.$

Here $\mathrm{p}_{0 \mathrm{~d}}(\mathrm{t})$ gives the probability that at time $\mathrm{t}$ all units are working, the repairman is preparing to take vacation and the system is working; $p_{0 v}(t, y)$ gives the probability that at time $t$ all units are working, the repairman is in vacation, the system is working and the elapsed vacation time lies in [y,y + dy); $\mathrm{p}_{1 \mathrm{v}}(\mathrm{t}, \mathrm{y})$ gives the probability that at time $\mathrm{t}$ one unit in the system is failed, the repairman is in vacation, the system is working and the elapsed vacation time lies in $[y, y+d y)$; $\mathrm{p}_{1 \mathrm{r}}(\mathrm{t}, \mathrm{x})$ represents the probability that at time $\mathrm{t}$ one unit in the system is failed, the failed unit being repaired, the system is working and the elapsed repair time lies in $[\mathrm{x}, \mathrm{x}+\mathrm{dx})$; $\mathrm{p}_{2 \mathrm{v}}(\mathrm{t}, \mathrm{y})$ gives the probability that at time $\mathrm{t}$ two units in the system are failed, the repairman is in vacation, the system is down and the elapsed vacation time lies in $[\mathrm{y}, \mathrm{y}+\mathrm{dy})$; $\mathrm{p}_{2 \mathrm{r}}(\mathrm{t}, \mathrm{x})$ represents the probability that at time $\mathrm{t}$ wo units in the system are failed, the one failed unit being repaired, another failed unit is waiting for repair, the system is down and and the elapsed repair time lies in $[\mathrm{x}, \mathrm{x}+\mathrm{dx}) ; \lambda, \lambda_{1}, \theta$ are positive constants; $\mu(\mathrm{x})$ is the repair rate function; $r(y)$ is the vacation rate function.

Throughout the paper we require the following assumption for the repair rate function; $\mu(\mathrm{x})$ and the vacation rate function $\mathrm{r}(\mathrm{y})$.

General Assumption 1.1: The functions $\mu(x)$ and $r(y)$ $: \mathrm{R}_{+} \rightarrow \mathrm{R}_{+}$are measurable and bounded such that

$$
\mu=\lim _{x \rightarrow \infty} \mu(x), r=\lim _{y \rightarrow \infty} r(y), \mu_{\infty}=\min (\mu, r) .
$$

\section{Problem as an Abstract Cauchy Problem}

To apply semigroup theory we transform in this section the system (GS), (BC), (IC) into an abstract Cauchy problem[6, DefП. 6.1] on the Banach space(X, \|. \|), where

$$
\mathrm{X}=\mathbb{C} \times \mathrm{L}_{\mathrm{y}}^{1}[0+\infty) \times \mathrm{L}_{\mathrm{y}}^{1}[0,+\infty) \times \mathrm{L}_{\mathrm{x}}^{1}[0,+\infty) \times \mathrm{L}_{\mathrm{y}}^{1}[0,+\infty)
$$




$$
\begin{aligned}
& \quad \times \mathrm{L}_{\mathrm{x}}^{1}[0,+\infty) \\
& \text { and }\|\mathrm{p}\|=\left|\mathrm{P}_{\mathrm{od}}\right|+\left\|\mathrm{P}_{\mathrm{ov}}\right\|_{L_{y}^{1}[0+\infty)}+\left\|\mathrm{P}_{1 \mathrm{v}}\right\|_{L_{y}^{1}[0+\infty)} \\
& \quad+\left\|\mathrm{P}_{1 \mathrm{r}}\right\|_{L_{x}^{1}[0,+\infty)}+\left\|\mathrm{P}_{2 \mathrm{v}}\right\|_{L_{y}^{1}[0+\infty)}+\left\|\mathrm{P}_{2 \mathrm{r}}\right\|_{L_{\mathrm{x}}^{1}[0,+\infty)}, \\
& \mathrm{p}=\left(\mathrm{p}_{0 \mathrm{~d}}, \mathrm{p}_{0 \mathrm{v}}(\mathrm{y}), \mathrm{p}_{1 \mathrm{v}}(\mathrm{y}), \mathrm{p}_{1 \mathrm{r}}(x), \mathrm{p}_{2 v}(\mathrm{y}), \mathrm{p}_{2 r}(\mathrm{x})\right)^{\mathrm{t}} \in X .
\end{aligned}
$$

To define the system operator $(A, D(A))$ we introduce a "maximal operator" $\left(A_{m}, D\left(A_{m}\right)\right)$ on $\mathrm{X}$ given as

$$
\begin{gathered}
A_{m}=\left(\begin{array}{cccccc}
-\left(N \lambda+\lambda_{1}+\theta\right) & \varphi_{1} & 0 & \varphi_{2} & 0 & 0 \\
0 & D_{1} & 0 & 0 & 0 & 0 \\
0 & \left(N \lambda+\lambda_{1}\right) & D_{2} & 0 & 0 & 0 \\
0 & 0 & 0 & D_{3} & 0 & 0 \\
0 & 0 & N \lambda & 0 & D_{4} & 0 \\
0 & 0 & 0 & N \lambda & 0 & D_{5}
\end{array}\right), \\
D\left(A_{m}\right)=\mathbb{C} \times \mathrm{W}_{\mathrm{y}}^{1,1}[0+\infty) \times \mathrm{W}_{\mathrm{y}}^{1,1}[0,+\infty) \times W_{\mathrm{x}}^{1,1}[0,+\infty) \\
\times \mathrm{W}_{\mathrm{y}}^{1,1}[0,+\infty) \times \mathrm{W}_{\mathrm{x}}^{1,1}[0,+\infty)
\end{gathered}
$$

where

$$
\begin{aligned}
& \varphi_{1}: L_{y}^{1}[0+\infty) \rightarrow \mathrm{C}, \mathrm{f} \rightarrow \varphi_{1}(f)=\int_{0}^{+\infty} r(y) f(y) d y \\
& \varphi_{1}: L_{x}^{1}[0+\infty) \rightarrow \mathrm{C}, \mathrm{g} \rightarrow \varphi_{2}(g)=\int_{0}^{+\infty} \mu(x) g(x) d x, \\
& D_{1}: W^{1,1}[0,+\infty) \rightarrow L^{1}[0,+\infty), \\
& f \rightarrow D_{1}(f)-\frac{d}{d y} f-\left(N \lambda+\lambda_{1}+r(y)\right) f, \\
& D_{2}: W^{1,1}[0,+\infty) \rightarrow L^{1}[0,+\infty), \\
& f \rightarrow D_{2}(f)=-\frac{d}{d y} f-(N \lambda+r(y)) f, \\
& D_{3}: W^{1,1}[0,+\infty) \rightarrow L^{1}[0,+\infty), \\
& f \rightarrow D_{3}(f)=-\frac{d}{d x} f-(N \lambda+\mu(x)) f, \\
& D_{4}: W^{1,1}[0,+\infty) \rightarrow L^{1}[0,+\infty), \\
& f \rightarrow D_{4}(f)=-\frac{d}{d y} f-r(y) f, \\
& D_{5}: W^{1,1}[0,+\infty) \rightarrow L^{1}[0,+\infty], \\
& f \rightarrow D_{5}(f)=-\frac{d}{d x} f-\mu(x) f,
\end{aligned}
$$

To model the boundary conditions $(B C)$ we use an abstract approach as in [7]. For this purpose we consider the "boundary space" $\partial X:=C^{2}$ and then define "boundary operators" $\mathrm{L}$ and $\Phi$ as follows.

$$
L: D\left(A_{m}\right) \rightarrow \partial X, \quad L\left(\begin{array}{c}
p_{0 d} \\
p_{0 v}(y) \\
p_{1 v}(y) \\
p_{1 r}(x) \\
p_{2 v}(y) \\
p_{2 r}(x)
\end{array}\right)=\left(\begin{array}{l}
p_{0 v}(0) \\
p_{1 v}(0) \\
p_{1 r}(0) \\
p_{2 v}(0) \\
p_{2 r}(0)
\end{array}\right)
$$

and $\phi: \mathrm{x} \rightarrow \partial \mathrm{x}$

$$
\Phi\left(\begin{array}{c}
p_{0 d} \\
p_{0 v}(y) \\
p_{1 v}(y) \\
p_{1 r}(x) \\
p_{2 v}(y) \\
p_{2 r}(x)
\end{array}\right)=\left(\begin{array}{cccccc}
\theta & 0 & 0 & 0 & 0 & 0 \\
0 & 0 & 0 & 0 & 0 & 0 \\
N \lambda+\lambda_{1} & 0 & \varphi_{1} & 0 & 0 & \varphi_{2} \\
0 & 0 & 0 & 0 & 0 & 0 \\
0 & 0 & 0 & 0 & \varphi_{1} & 0
\end{array}\right)\left(\begin{array}{c}
p_{0 d} \\
p_{0 v}(y) \\
p_{1 v}(y) \\
p_{1 r}(x) \\
p_{2 v}(y) \\
p_{2 r}(x)
\end{array}\right)
$$

If the operator $(A, D(A))$ on $\mathrm{X}$ is then defined as

$$
A p=A_{m} p, D(A)=\left\{p \in D\left(A_{m}\right) \mid \Leftrightarrow L p=\Phi p\right\},
$$

then the model of the system $(G S),(B C)$ and (IC) can be described equivalently to the abstract Cauchy problem as follows.

$$
\left\{\begin{array}{l}
\frac{d p(t)}{d t}=A p(t), \quad t \in[0,+\infty), \\
p(0)=(1,0,0,0,0,0)^{T} \in X .
\end{array}\right.
$$

\section{CHARACTERISTIC EQUATION}

In this section we characterize $\delta(A)$ by the spectrum of a scalar $5 \times 5$-matrix, i.e., or we obtain a characteristic equation which relates $\delta(A)$ to the spectrum of an operator on the boundary space $\partial \mathrm{X}$. For this purpose, we apply techniques and results from [8]. We start from the operator $\left(A_{0}, D\left(A_{0}\right)\right)$ defined by

$$
D\left(A_{0}\right)=\left\{p \in D\left(A_{m}\right) \mid L p=0\right\}, A_{0} p=A_{m} p
$$

The elements in $\operatorname{ker}\left(\gamma-A_{m}\right)$ can be expressed as follows

Lemma 3.1: For $\gamma \in\left(A_{0}\right)$, we have

$$
\begin{aligned}
& \mathrm{p} \in \operatorname{ker}\left(\gamma-A_{m}\right) \quad \mathrm{p}= \\
& \left(\mathrm{p}_{0 \mathrm{~d}}, \mathrm{p}_{0 \mathrm{v}}(\mathrm{y}), \mathrm{p}_{1 \mathrm{v}}(\mathrm{y}), \mathrm{p}_{1 \mathrm{r}}(x), \mathrm{p}_{2 v}(\mathrm{y}), \mathrm{p}_{2 r}(\mathrm{x})\right)^{\mathrm{t}} \in D\left(A_{m}\right) \text { with } \\
& p_{0 v}(y)=a_{1} e^{-\left(\gamma+\lambda N+\lambda_{1}\right) y-\int_{0}^{+\infty} r(y) d \tau} ; \\
& p_{1 v}(y)=a_{1} \frac{N \lambda+\lambda_{1}}{\lambda_{1}}\left(1-e^{-\lambda_{1} y}\right) e^{-(\gamma+N \lambda) y-\int_{0}^{y} r(\tau) d \tau} \\
& +a_{2} e^{-(\gamma+N \lambda)-\int_{0}^{y} r(\tau) d \tau} \\
& p_{1 r}(x)=a_{3} e^{-(\gamma+N \lambda) x-\int_{0}^{x} \mu(\tau) d \tau} \quad ; \\
& p_{2 v}(y)=\frac{a_{1}}{\lambda_{1}} e^{-\gamma y-\int_{0}^{y} r(\tau) d \tau}\left[\left(N \lambda+\lambda_{1}\right)\left(1-e^{-N \lambda y}\right)-\right. \\
& \left.N \lambda\left(1-e^{-\left(N \lambda+\lambda_{1}\right) y}\right)\right] \\
& +a_{2} e^{-\gamma y-\int_{0}^{y} r(\tau) d \tau}\left(1-e^{-N \lambda y}\right)+a_{4} e^{-\gamma y-\int_{0}^{y} r(\tau) d \tau} ; \\
& p_{2 r}(x)=a_{3} e^{-\gamma x-\int_{0}^{x} \mu(\tau) d \tau}\left(1-e^{-N \lambda x}\right)+a_{5} e^{-\gamma x-\int_{0}^{x} \mu(\tau) d \tau} ;
\end{aligned}
$$

Using $\left[8\right.$, Lemma1.2] the domain $\mathrm{D}\left(A_{m}\right)$ of the maximal operator $A_{m}$ decomposes as

$$
\mathrm{D}\left(A_{m}\right)=\mathrm{D}\left(A_{0}\right) \oplus \operatorname{ker}\left(\gamma-A_{m}\right) .
$$

Moreover, since $\mathrm{L}$ is surjective, $\left.\mathrm{L}\right|_{\operatorname{ker}\left(\gamma-A_{m}\right)}:\left(\gamma-A_{m}\right) \rightarrow$ $\partial X$ is invertible for each $\gamma \in \rho\left(A_{0}\right)$, see[8,Lemma1.2]. We denote its inverse by 


$$
D_{\gamma}:=\left(\left.L\right|_{k e r\left(\gamma-A_{m}\right)}\right)^{-1}: \partial X \rightarrow \operatorname{ker}\left(\gamma-A_{m}\right)
$$

and call it "Dirichlet operator".

We can give the explicit form of $D_{\gamma}$ as follows.

Lemma 3.2: For each $\gamma \in \rho\left(A_{0}\right)$, the Dirichlet operator $\mathrm{D}_{\gamma}$ has the form

$$
D_{\gamma}=\left(\begin{array}{ccccc}
D_{11} & 0 & D_{13} & 0 & 0 \\
D_{21} & 0 & 0 & 0 & 0 \\
D_{31} & D_{32} & 0 & 0 & 0 \\
0 & 0 & D_{43} & 0 & 0 \\
D_{51} & D_{52} & 0 & D_{54} & 0 \\
0 & 0 & D_{63} & 0 & D_{65}
\end{array}\right),
$$

where

$$
\begin{aligned}
D_{11}= & \frac{1}{\gamma+N \lambda+\lambda_{1+\theta}} \int_{0}^{+\infty} r(y) e^{-\left(\gamma+N \lambda+\lambda_{1}\right) y-\int_{0}^{y} r(\tau) d \tau} d y ; \\
D_{13}= & \frac{1}{\gamma+N \lambda+\lambda_{1}+\theta} \int_{0}^{+\infty} \mu(x) e^{-(\gamma+N \lambda) x-\int_{0}^{x} \mu(\tau) d \tau} ; \\
D_{21}= & e^{-\left(\gamma+N \lambda+\lambda_{1}\right) y-\int_{0}^{y} r(\tau) d \tau} ; \\
D_{31}= & \frac{N \lambda+\lambda_{1}}{\lambda_{1}}\left(1-e^{-\lambda_{1} y}\right) e^{-(\gamma+N \lambda) y-\int_{0}^{y} r(\tau) d \tau} ; \\
D_{32}= & e^{-(\gamma+N \lambda) y-\int_{0}^{y} r(\tau) d \tau} ; \\
D_{43}= & e^{-(\gamma+N \lambda) x-\int_{0}^{x} \mu(\tau) d \tau} ; \\
D_{51}= & \frac{1}{\lambda_{1}}\left[\left(N \lambda+\lambda_{1}\right)\left(1-e^{-N \lambda y}\right)-N \lambda\left(1-e^{-\left(N \lambda+\lambda_{1}\right) y}\right)\right] \\
& \times e^{-\gamma y-\int_{0}^{y} r(\tau) d \tau} \\
D_{52}= & \left(1-e^{-N \lambda y}\right) e^{-\gamma y-\int_{0}^{y} r(\tau) d \tau} ; D_{54}=e^{-\gamma y-\int_{0}^{y} r(\tau) d \tau ;} \\
D_{63}= & \left(1-e^{-N \lambda x}\right) e^{-\gamma x-\int_{0}^{x} \mu(\tau) d \tau} ; D_{65}=e^{-\gamma x-\int_{0}^{x} \mu(\tau) d \tau} ;
\end{aligned}
$$

For $\gamma \in \rho\left(A_{0}\right)$, the operator $\Phi D_{\gamma}$ can be represented by the $5 \times 5$-matrix

$$
\phi D_{\gamma}=\left(\begin{array}{ccccc}
a_{11} & 0 & a_{13} & 0 & 0 \\
0 & 0 & 0 & 0 & 0 \\
a_{31} & a_{32} & a_{33} & 0 & a_{35} \\
0 & 0 & 0 & 0 & 0 \\
a_{51} & a_{52} & 0 & a_{54} & 0
\end{array}\right)
$$

where

$$
\begin{aligned}
a_{11}= & \frac{\theta}{\gamma+N \lambda+\lambda_{1}+\theta} \int_{0}^{+\infty} r(y) e^{-\left(\gamma+N \lambda+\lambda_{1}\right) y-\int_{0}^{y} r(\tau) d \tau} d y ; \\
a_{13}= & \frac{\theta}{\gamma+N \lambda+\lambda_{1}+\theta} \int_{0}^{+\infty} \mu(x) e^{-(\gamma+N \lambda) x-\int_{0}^{x} \mu(\tau) d \tau} d x ; \\
a_{31}= & \frac{N \lambda+\lambda_{1}}{\gamma+N \lambda+\lambda_{1}+\theta} \int_{0}^{+\infty} r(y) e^{-\left(\gamma+N \lambda+\lambda_{1}\right) y-\int_{0}^{y} r(\tau) d \tau} d y+ \\
& \frac{\left(N \lambda+\lambda_{1}\right)}{\lambda_{1}} \int_{0}^{+\infty} r(y)\left(1-e^{-\lambda_{1} y}\right) e^{-(\gamma+N \lambda) y-\int_{0}^{y} r(\tau) d \tau} d y ; \\
a_{32}= & \int_{0}^{+\infty} r(y) e^{-(\gamma+N \lambda) y-\int_{0}^{y} \mu(\tau) d \tau} d y
\end{aligned}
$$

$$
\begin{aligned}
& a_{33}=\frac{N \lambda+\lambda_{1}}{\gamma+N \lambda+\lambda_{1}+\theta} \int_{0}^{+\infty} \mu(x) e^{-(\gamma+N \lambda) x-\int_{0}^{x} \mu(\tau) d \tau} d x \\
& +\int_{0}^{+\infty} \mu(x)\left(1-e^{-N \lambda x}\right) e^{-\gamma x-\int_{0}^{x} \mu(\tau) d \tau} d x ; \\
& a_{35}=\int_{0}^{+\infty} \mu(x) e^{-\gamma x-\int_{0}^{x} \mu(\tau) d \tau} d x ; \\
& a_{51}=\frac{N \lambda+\lambda_{1}}{\lambda_{1}} \int_{0}^{+\infty}\left(1-e^{-N \lambda y}\right) e^{-\gamma y-\int_{0}^{y} r(\tau) d \tau} r(y) d y \\
& -\frac{N \lambda}{\lambda_{1}} \int_{0}^{+\infty} r(y)\left(1-e^{-\left(N \lambda+\lambda_{1}\right) y}\right) e^{-\gamma y-\int_{0}^{y} \mu(\tau) d \tau} d y \\
& a_{52}=\int_{0}^{+\infty} r(y)\left(1-e^{-N \lambda y}\right) e^{-\gamma y-\int_{0}^{y} r(\tau) d \tau} d y \quad ; \\
& a_{54}=\int_{0}^{+\infty} r(y) e^{-\gamma x-\int_{0}^{y} r(\tau) d \tau} d y ;
\end{aligned}
$$

The Following result, which can be found in [9], plays important role for us to prove the well-posedness of the system.

Lemma 3.3(The characteristic equation): If $\gamma \in \mathrm{p}\left(A_{0}\right)$ and there exists $\gamma_{0} \in \mathrm{C}$ such that $1 \notin \sigma\left(\Phi D_{r}\right)$, then

$$
\gamma \in \sigma(\mathrm{A}) \Leftrightarrow 1 \in \sigma\left(\Phi D_{\gamma}\right) \text {. }
$$

\section{WELL-POSEDNESS OF THE SYSTEM}

Our main goal in this section is to prove the well-posedness of the system. From this we obtain the existence of a unique positive dynamic solution of the system. We first prove that the operator A generates a positive contraction $\mathrm{C}_{0}$-semigroup $(T(t))_{t \geq 0}$. For this purpose we will check that operator A fulfills all the conditions in the Phillips' theorem, see [10, Thm.C_П1.2]. The following lemma shows the surjectivity of $\gamma-A$ for $\gamma>0$.

Lemma 4.1: If $\gamma \in R, \gamma>0$, then $\gamma \in p(A)$. ,

Proof: Let $\gamma \in R, \gamma>0$. Then all the entries of $\Phi \mathrm{D}_{\gamma}$ are positive and using only elementary calculations one can show that both column sums are strictly less than 1 . Hence, $\left\|\Phi D_{7}\right\|$ $<1$ and thus $1 \notin \sigma\left(\Phi D_{7}\right)$. Using Lemma 3.3 we conclude that $\in p(A)$.

Lemma 4.2: $A: D(A) \rightarrow R(A) \subset X \quad$ is a closed linear operator and $D(A)$ is dense in $\mathrm{X}$.

If $X^{\prime}$ denotes the dual space of $X$, then $X^{\prime}=C \times\left(L_{y}^{\infty}[0,+\infty)\right)^{2} \times L_{x}^{\infty}[0,+\infty) \times L_{y}^{\infty}[0,+\infty) \times$ $L_{x}^{\infty}[0,+\infty)$. norm

It is obvious that $X^{\prime}$ is a Banach space endowed with the $\|q\|=\max \left(\left|q_{0 d}\right|,\left\|q_{0 v}\right\|_{L_{y}^{\infty}[0,+\infty)},\left\|q_{1 v}\right\|_{L_{y}^{\infty}[0,+\infty)},\left\|q_{1 r}\right\|_{L_{x}^{\infty}[0,+\infty)}\right.$,

$$
\left.\left\|q_{2 v}\right\|_{L_{y}^{\infty}[0,+\infty)},\left\|q_{2 r}\right\|_{L_{x}^{\infty}[0,+\infty)}\right)
$$

where $\mathrm{q}=\left(q_{0 d}, q_{0 v}(y), q_{1 v}(y), q_{1 r}(x), q_{2 v}(y), q_{2 r}(x)\right)^{t} \in X^{\prime}$.

Lemma 4.3: The operator $(A, D(A))$ is dispersive.

Proof: For $p=\left(p_{0 d}, p_{0 v}(y), p_{1 v}(y), p_{1 r}(x), p_{2 v}(y), p_{2 r}(x)\right)^{t} \in$ $X$, we define 


$$
q=\left(q_{0 d}, q_{0 v}(y), q_{1 v}(y), q_{1 r}(x), q_{2 v}(y), q_{2 r}(x)\right)^{t} \in X^{\prime},
$$

where

$$
\begin{gathered}
q_{o d}=\|p\| s g n_{+}\left(p_{o d}\right) ; q_{i v}(y)=\|p\| s g n_{+} p_{i v}(y), \\
i=o, 1,2 ; \\
q_{j r}(x)=\|p\| s g n_{+} p_{j r}(y), j=12 ; \\
\operatorname{sgn}_{+}\left(p_{o d}\right)=\left\{\begin{array}{ll}
1, & p_{o d}>0 \\
0, & p_{o d} \leq 0
\end{array} ;\right. \\
\operatorname{sgn}_{+} p_{i v}(y)=\left\{\begin{array}{ll}
1, & p_{i v}(y)>0 \\
0, & p_{i v}(y) \leq 0,
\end{array} \quad i=0,1,2 ;\right. \\
\operatorname{sgn}_{+} p_{j r}(x)=\left\{\begin{array}{ll}
1, & p_{j r}(x)>0 \\
0, & p_{j r}(x) \leq 0,
\end{array} \quad j=1,2 ;\right.
\end{gathered}
$$

Noting the boundary condition, it is not difficult to prove that $\langle A p, q\rangle \leq 0$. By $[6$, p. 49] we obtain that $(A, D(A))$ is a dispersive operator.

From Lemma 4.1- 4.3 we see that all the conditions in Phillips' theorem (see[10, Thm. C_ח1.2] ) are fulfilled and thus we obtain the following result.

Theorem 4.4: The operator $(A, D(A))$ generates a positive contraction $\mathrm{C}_{0}$-semigroup $(\mathrm{T}(\mathrm{t}))_{\mathrm{t} \geq 0}$.

From Theorem 4.4 and [6, Cor.II.6.9] we can characterize the well-Posedness of $(A C P)$ as follows.

Theorem 4.5: The associated abstract Cauchy problem (ACP) is well-posed.

From Theorem 4.5 and [6, Prop. II. 6.2] we can state our main result.

Theorem 4.6: The system (R), (BC) and (IC) has a unique positive dynamic solution

$p(t)=\left(p_{0 d}, p_{0 v}(t, y), p_{1 v}(t, y), p_{1 r}(t, x), p_{2 v}(t, y), p_{2 r}(t, x)\right)^{t} \in X$ which satisfies $\|p(t)\|=1, t \in[0,+\infty)$.

\section{ACKNOWLEDGMENT}

This work was supported by the National Natural Science Foundation of China (No.11361057) and the National Natural Science Foundation of Xinjiang Uighur Autonomous Region (No. 2014211A002).

\section{REFERENCES}

[1] Gnedenko, B.V.: Some theorems on standbys. In: Proc. Fifth Berkeley Sympos. Mathematical Statistics and Probability, Berkeley, Calif., 1965/66. Physical Sciences, University California Press, Berkeley 1967, vol. III, pp. 285-291.

[2] Subramanian, R.: Availability of a Gnedenko system. IEEE Trans. Reliab. , 1977, 26, pp. 302-303.

[3] Cao, J.: Availability and failure frequency of a Gnedenko system. Ann. Oper. Res. , 1990,24, pp. 55-68.

[4] Cao, J., Yue, D.: The Gnedenko system attended by a repairman with single vacations. J. Shandong Univ. Nat. Sci., 2008, 43(6), pp. 77-82.

[5] Guo, W. L., Wu, Q. T., The Gnedenko system attended by a repairman with multiple delay vacations, Shandong Jianzhu Univ. Nat. Sci. 2009, 24(4), 342-345.
[6] Engel K.-J., Nagel R., One-Parameter Semigroups for Linear Evolution Equations. Graduate Texts in Mathematics, 194, Springer-Verlag, 2000.

[7] Casarino V., Engel K.-J., R. Nagel and Nickel G. (2003) A semigroup approach to boundary feedback systems. Integr. Equ. Oper. Theory, 47, 289-306.

[8] Greiner G. (1987) Perturbing the boundary conditions of a generator. Houston J. Math., 13, 213-229.

[9] Haji A. and Radl A. (2007) A semigroup approach to queueing systems. Semigroup Forum, 75, 610-624.

[10] Nagel R. (1986) One-parameter Semigroups of Positive Operators. Springer-Verlag. 